\title{
RELATIVE STABILITY AND WEAK CONVERGENCE IN NON-DECREASING STOCHASTICALLY MONOTONE MARKOV CHAINS ${ }^{1}$
}

\author{
P. TODOROVIC \\ University of California \\ Department of Statistics and Applied Probability \\ Santa Barbara, CA
}

\begin{abstract}
Let $\left\{\xi_{n}\right\}$ be a non-decreasing stochastically monotone Markov chain whose transition probability $Q(\cdot, \cdot)$ has $Q(x,\{x\})=\beta(x)>0$ for some function $\beta(\cdot)$ that is non-decreasing with $\beta(x) \uparrow 1$ as $x \rightarrow+\infty$, and each $Q(x, \cdot)$ is non-atomic otherwise. A typical realization of $\left\{\xi_{n}\right\}$ is a Markov renewal process $\left\{\left(X_{n}, T_{n}\right)\right\}$, where $\xi_{j}=X_{n}$ for $T_{n}$ consecutive values of $j, T_{n}$ geometric on $\{1,2, \ldots\}$ with parameter $\beta\left(X_{n}\right)$. Conditions are given for $X_{n}$ to be relatively stable and for $T_{n}$ to be weakly convergent.
\end{abstract}

Key words: Markov chain, stochastic monotonicity, Markov renewal process, relative stability, weak convergence.

AMS (MOS) subject classifications:60G, 60K15.

\section{INTRODUCTION}

In this paper, $R$ is the real line and $\Re_{B}$ the $\sigma$-field of Borel subsets of $R$. Let $\left\{\xi_{n}\right\}_{0}^{\infty}$ be a Markov chain with state space $\left\{R, \Re_{B}\right\}$, an initial distribution $\pi$ and transition probability $Q$. The $\pi$ and $Q$ determine completely and uniquely a probability measure $P$ on the countable product space $\left\{R^{\infty}, \Re^{\infty}\right\}$. When $\pi(\cdot)=\epsilon_{x}(\cdot)$ (the Dirac measure concentrated at $x$ ) we shall write $P_{x}$ instead of $P$. The corresponding expectation operator is denoted then by $E_{x}$.

Throughout this paper it is assumed that the $Q$ is subject to the following regularity conditions:

(i) for each $x \in R$ the support of $Q(x, \cdot)$ is in $[x, \infty)$ :

(ii) the chain $\left\{\xi_{n}\right\}_{0}^{\infty}$ is stochastically monotone (Daley, [3]); in other words; for any $x_{1} \leq x_{2}, Q\left(x_{2}, B_{y}\right) \leq Q\left(x_{1}, B_{y}\right)$ where $B_{y}=(-\infty, y]$

$$
Q(x,\{y\})= \begin{cases}0 & x \neq y \\ \beta(x)>0 & x=y\end{cases}
$$

\footnotetext{
${ }^{1}$ Received: January, 1991. Revised: June, 1991.
} 
Concerning the function $\beta(\cdot)$ we assume that $\left(x_{1} \leq x_{2}\right)$

$$
\beta\left(x_{1}\right) \leq \beta\left(x_{2}\right) \text { and } \lim _{x \rightarrow+\infty} \beta(x)=1
$$

From (1.1.i) it follows that

$$
\xi_{0} \leq \xi_{1} \leq \ldots
$$

Markov processes of this type are of considerable interest in reliability theory as models of the amount of deterioration of a mechanical device subject to shocks and wear during its service (Barlow and Proschan, [1]; Brown and Changanty, [2]).

Set

$$
\begin{gathered}
\tau_{0}=\sup \left\{k ; \xi_{k}=\xi_{0}\right\}, \tau_{n}=\sup \left\{k ; \xi_{k}=\xi_{\tau_{n-1}+1}\right\}, T_{0}=\tau_{0} \\
T_{n}=\tau_{n}-\tau_{n-1}, X_{0}=\xi_{0}, X_{n}=\xi_{\tau_{n-1}+1}, W_{0}=X_{0}, W_{n}=X_{n}-X_{n-1} .
\end{gathered}
$$

From this one readily obtains that

$$
P_{x}\left\{T_{0} \geq i\right\}=\{\beta(x)\}^{i} \quad i=0,1, \ldots
$$

\section{AUXILIARY RESULTS}

Here we list some basic properties of the bivariate sequence $\left\{\left(X_{n}, T_{n}\right)\right\}$ needed in the rest of this paper. Some simple calculations show that this sequence is a Markov renewal process with the transition probability

$$
P\left\{X_{n} \in d u, T_{n}=i \mid X_{n-1}\right\}=P\left(X_{n-1}, d u\right)\{1-\beta(u)\}\{\beta(u)\}^{i-1} \quad(\text { a.s. })
$$

where $i=1,2, \ldots$ and

$$
P\left(x, B_{y}\right)= \begin{cases}0 & y<x \\ \frac{Q\left(x, B_{y}\right)-\beta(x)}{1-\beta(x)} & y \geq x .\end{cases}
$$

The $P\left(x, B_{y}\right)$ is the transition probability of the Markov chain $\left\{X_{n}\right\}_{0}^{\infty}$. It is easy to verify that

$$
P\left(x_{2}, B y\right) \leq P\left(x_{1}, B_{y}\right) \text { for all } x_{1} \leq x_{2}
$$

From (2.1) we deduce

$$
P\left\{X_{n} \in d u, T_{n}=i\right\}=\{1-\beta(u)\}\{\beta(u)\}^{i-1} P\left\{X_{n} \in d u\right\}
$$

which clearly implies (see (1.5)) that 


$$
\begin{aligned}
P\left\{T_{n}=i \mid X_{n}\right\} & =\left\{1-\beta\left(X_{n}\right)\right\}\left\{\beta\left(X_{n}\right)\right\}^{i-1} \text { (a.s.) } \\
& =P_{X_{n}}\left\{T_{0}=i-1\right\} .
\end{aligned}
$$

In addition, since

$$
P_{x}\left\{X_{1} \in d u, T_{1}=i\right\}=P(x, d u)\{1-\beta(u)\}\{\beta(u)\}^{i-1}
$$

it follows that

$$
P\left\{X_{n} \in d u, T_{n}=i \mid X_{n-1}\right\}=P_{X_{n-1}}\left\{X_{1} \in d u, T_{1}=j\right\} \text { (a.s.). }
$$

Denote by $P^{n}\left(x, B_{y}\right)$ the $n$-step transition probability of $\left\{X_{n}\right\}_{0}^{\infty}$, since

$$
X_{0}<X_{1}<\ldots
$$

we have that $P^{n+1}\left(x, B_{y}\right) \leq P^{n}\left(x, B_{y}\right)$. On the other hand, the stochastic monotonicity and the Chapman-Kolmogorov equation yield:

$$
P^{n}\left(x, B_{y}\right) \leq\left(P\left(x, B_{y}\right)\right)^{n} .
$$

From this, (2.8) and the Borel-Cantelli lemma it follows that $X_{n} \rightarrow+\infty(a . s$.$) if P\left(x, B_{y}\right)<1$ for all $y<\infty$.

It is clear from (2.5) that $T_{n}$ is conditionally geometric with parameter $\beta\left(X_{n}\right)$. In addition, since

$$
P\left\{T_{n} \geq i \mid X_{n}\right\}=\left\{\beta\left(X_{n}\right)\right\}^{i-1} \text { (a.s.) }
$$

it is apparent that $\left\{T_{n}\right\}$ is stochastically monotone and that $T_{n} \underset{n}{d . \infty}$ as $n \rightarrow \infty$. Finally, for each $n=0,1, \ldots$ we have:

$$
P\left\{T_{0}=i_{0}, \ldots, T_{n}=i_{n}\left|X_{0}, \ldots, X_{n}\right|=\prod_{j=1}^{n} P\left\{T_{j}=i_{j} \mid X_{j}\right\} \quad\right. \text { (a.s.). }
$$

In other words, conditioned on a realization of $\left\{X_{n}\right\}$ the sequence of sojourn times $\left\{T_{n}\right\}$ becomes a family of independent $r . v$.'s such that the distribution of $T_{n}$ depends only on $X_{n}$.

Consider

$$
\begin{aligned}
P_{x}\left\{X_{n} \in d u, T_{n}\right. & =i\}=\int_{x}^{u} P\left\{X_{n} \in d u, T_{n}=i \mid X_{n-1}=z\right\} P^{n-1}(x, d z) \\
& =\{1-\beta(u)\}\{\beta(u)\}^{i-1} P_{x}\left\{X_{n} \in d u\right\}
\end{aligned}
$$


from which we deduce that

$$
P_{x}\left\{T_{n}=i \mid X_{n}\right\}=\left\{1-\beta\left(X_{n}\right)\right\}\left\{\beta\left(X_{n}\right)\right\}^{i-1}(\text { a.s. })
$$

where the right hand side is independent of $x$.

\section{REMARKS ON THE STRUCTURE OF $\left\{W_{n}\right\}$}

In this section we investigate asymptotic structure of the sequence $\left\{W_{n}\right\}_{0}^{\infty}$ assuming that the following condition holds for all $y \geq 0$ :

$$
\lim _{x \rightarrow \infty} \frac{P_{x}\left\{\xi_{1}>x+y\right\}}{P_{x}\left\{\xi_{1}>x\right\}}=1-F(y)
$$

where $F(\cdot)$ is a proper $d . f$.

Remark 3.1: The condition (3.1) is similar to one introduced by Gnedenko [4].

Denote by

$$
\Phi_{n}(y \mid x)=P_{x}\left\{W_{n} \leq y\right\} \quad n=1,2, \ldots
$$

then clearly

$$
\Phi_{1}(y \mid x)=P\left(x, B_{x+y}\right)
$$

Some simple calculations yield:

$$
\Phi_{n}(y \mid x)=E_{x}\left\{\Phi_{1}\left(y \mid X_{n-1}\right)\right\} .
$$

Taking into account (2.2) and condition (3.1), we have:

$$
\lim _{x \rightarrow \infty} \Phi_{1}(y \mid x)=F(y)
$$

This, (3.4) and the Lebesgue bounded convergence theorem imply that

$$
\lim _{n \rightarrow \infty} \Phi_{n}(y \mid x)=F(y)
$$

In other words, (at least) $W_{n}^{d .} \rightarrow$, where $Y$ is a r.v. with the $d . f . F(y)$. The following proposition generalizes this simple observation.

Proposition 3.1: Assume that (3.1) holds and $F(\cdot)$ is continuous, then under $P_{x}$, for all $k=1,2, \ldots$

$$
\left(W_{n+1}, \ldots, W_{n+k}\right) \stackrel{d}{\rightarrow}\left(Y_{1}, \ldots, Y_{k}\right) \text { as } n \rightarrow \infty
$$

where $\left\{Y_{i}\right\}_{1}^{\infty}$ is an i.i.d. sequence of r.v.'s with common d.f. $F(\cdot)$. 
Proof: The method of proof will be amply illustrated by the case $n=2$. Given $\epsilon>0$, we obtain

$$
\begin{gathered}
P_{x}\left\{W_{n+1} \leq y_{1}, W_{n+2} \leq y_{2}\right\} \\
=\int_{x}^{\infty} \int_{z}^{z+y_{1}} P(z, d u) \Phi_{1}\left(y_{2} \mid u\right) P^{n}(x, d z) \\
=E_{x}\left\{\int_{X_{n}}^{X_{n}+y_{1}} P\left(X_{n}, d u\right) \Phi_{1}\left(y_{2} \mid u\right)\right\}
\end{gathered}
$$

Since by assumption $F(\cdot)$ is continuous the convergence in (3.5) is uniform. Consequently, for any $\epsilon>0$ there exists $x_{0}$ such that

$$
\left|\Phi_{1}\left(y_{2} \mid u\right)-F\left(y_{2}\right)\right|<\epsilon \text { for all } u>x_{0} \text { and any } y_{2} \in R \text {. }
$$

From this and (3.8) we then have:

$$
\begin{gathered}
P_{x}\left\{W_{n+1} \leq y_{1}, W_{n+2} \leq y_{2}\right\} \leq P^{n}\left(x, B_{x_{0}}\right) \\
+E_{x}\left\{I_{\left\{X_{n}>x_{0}\right\}} \int_{X_{n}}^{X_{n}+y_{1}} \Phi_{1}\left(y_{2} \mid u\right) P\left(X_{n}, d u\right)\right\} \\
\leq P^{n}\left(x, B_{x_{0}}\right)+\left(\epsilon+F\left(y_{2}\right)\right) E_{x}\left\{\Phi_{1}\left(y_{1} \mid X_{n}\right) I_{\left\{X_{n}>x_{0}\right\}}\right\} .
\end{gathered}
$$

Consequently,

$$
\varlimsup_{n \rightarrow \infty} P_{x}\left\{W_{n+1} \leq y_{1}, W_{n+2} \leq y_{2}\right\} \leq\left(\epsilon+F\left(y_{2}\right)\right) F\left(y_{1}\right) .
$$

In the same fashion, one can show that

$$
\lim _{n \rightarrow \infty} P_{x}\left\{W_{n+1} \leq y_{1}, W_{n+2} \leq y_{2}\right\} \geq F\left(y_{1}\right)\left(F\left(y_{2}\right)-\epsilon\right) .
$$

Since $\epsilon>0$ is arbitrary, the assertion follows.

Remark 3.2: The last proposition indicates that, roughly speaking, the remote members of $\left\{W_{n}\right\}_{0}^{\infty}$ are i.i.d. r.v.'s.

Next, we show that the sequence $\left\{W_{n}\right\}_{0}^{\infty}$ is endowed with a mixing property, which means, loosely speaking, that its elements far apart are nearly independent. Denote by $\mathcal{F}_{n}=\sigma\left\{W_{0}, \ldots, W_{n}\right\}$ and by $\mathscr{F}^{n}=\sigma\left\{W_{n}, W_{n+1} \ldots\right\}$ then we have:

Proposition 3.2: For each $n=1,2, \ldots$ and $k=1,2, \ldots$ 


$$
\begin{aligned}
& \lim _{m \rightarrow \infty} P_{x}\left(\bigcap_{j=1}^{n}\left\{X_{j} \leq y_{j}\right\} \bigcap_{i=1}^{k}\left\{W_{n+m+i} \leq z_{i}\right\}\right) \\
= & P_{x}\left(\bigcap_{j=1}^{n}\left\{X_{j} \leq y_{j}\right\}\right) \prod_{i=1}^{k} F\left(z_{i}\right) \quad\left(x<y_{1}<\ldots<y_{n}\right)
\end{aligned}
$$

Proof: By invoking the Markov property of $\left\{X_{n}\right\}_{0}^{\infty}$ and the proposition 3.1, we obtain

$$
\begin{gathered}
P_{x}\left(\bigcap_{j=1}^{n}\left\{X_{j} \leq y_{j}\right\} \bigcap_{i=1}^{k}\left\{W_{n+m+i} \leq z_{i}\right\}\right) \\
=\int_{\left(x, y_{n}\right]} P_{x}\left(\bigcap_{j=1}^{n}\left\{X_{j} \leq y_{j}\right\} \mid X_{n}=s\right) P_{s}\left(\bigcap_{i=1}^{k}\left\{W_{m+i} \leq z_{i}\right\}\right) P^{n}(x, d s) \\
\rightarrow \int_{\left(x, y_{n}\right]} P_{x}\left(\bigcap_{j=1}^{n}\left\{X_{j} \leq y_{j}\right\} \mid X_{n}=s\right) \prod_{i=1}^{k} F\left(z_{i}\right) P^{n}(x, d s)
\end{gathered}
$$

as $m \rightarrow \infty$ which proves the assertion.

Corollary 3.1: $\quad\left\{W_{n}\right\}_{0}^{\infty}$ and $\left\{Y_{j}\right\}_{1}^{\infty}$ are independent families. Set

$$
\sigma=\bigcap_{n=0}^{\infty} \mathscr{F}^{n} .
$$

It follows from the last proposition that $\mathcal{F}_{n}$ and $\mathcal{T}$ are independent $\sigma$-algebras for all $n=0,1, \ldots$. Therefore $\mathcal{F}_{n} \cap \mathcal{T}$ is a trivial $\sigma$-algebra (its elements are either sure or null events). By letting $n \rightarrow \infty$ we have that $\digamma_{\infty} \supset \mathcal{\sigma}$ and that their intersection is a trivial $\sigma$ algebra. This clearly implies that the tail $\sigma$-algebra $\mathscr{T}$ is a trivial one.

\section{RELATIVE STABILITY OF $\left\{X_{n}\right\}$}

The sequence $\left\{X_{n}\right\}$ is said to be relatively stable if there exist constants $\left\{a_{n}\right\}$ such that $X_{n} / a_{n} \rightarrow 1$ in probability (Gnedenko and Kolmogorov, [5]). If the convergence is (a.s.) the sequence is called (a.s.) relatively stable (Resnik, [6]). The following proposition gives a sufficient condition for (a.s.) relative stability of $\left\{X_{n}\right\}$.

Proposition 4.1: Assume that

$$
\sup _{x} E_{x}\left\{W_{1}^{2}\right\}<\infty
$$

then $X_{n} / n \rightarrow \alpha_{1}$ (a.s.) where $\alpha_{1}=E\left\{Y_{1}\right\}$.

Proof: $\quad$ From (3.4), (4.1) and Fubini's theorem we deduce that 


$$
\begin{gathered}
E_{x}\left\{W_{k}^{2}\right\}=\int_{0}^{\infty} y\left[1-\Phi_{k}(y \mid x)\right] d y \\
=E_{x}\left\{\int_{0}^{\infty} y\left[1-\Phi_{1}\left(y \mid X_{k-1}\right)\right] d y\right\} \\
=E_{x}\left(E_{X_{k-1}}\left\{W_{1}^{2}\right\}\right) \leq \sup _{x} E_{x}\left\{W_{1}^{2}\right\}<\infty .
\end{gathered}
$$

Consequently,

$$
\sup _{x, k} E_{x}\left\{W_{k}^{2}\right\}<\infty
$$

Next, since

$$
\left\{\frac{1}{n} \sum_{1}^{n} W_{k} \text { converges }\right\} \in \mathcal{T}
$$

and $\mathcal{T}$ is a trivial $\sigma$-algebra, to prove the proposition it suffices to show that

$$
P_{x}\left\{\left|\frac{1}{n} X_{n}-\alpha_{1}\right|>\epsilon\right\} \rightarrow 0 \text { as } n \rightarrow \infty
$$

Consider

$$
\operatorname{Var}\left\{\frac{1}{n} X_{n}\right\}=\frac{1}{n^{2}}\left(\sum_{k=1}^{n} \operatorname{Var}\left\{W_{k}\right\}+\sum_{i=1}^{n-1} \sum_{j=i+1}^{n} \operatorname{Cov}\left(W_{i}, W_{j}\right)\right) .
$$

It is clear from (4.1) and (4.2) that under $P_{x}$

$$
\frac{1}{n^{2}} \sum_{k=1}^{n} \operatorname{Var}\left\{W_{k}\right\} \rightarrow 0 \text { as } n \rightarrow \infty \text {. }
$$

On the other hand, due to propositions 3.1 and 3.2

$$
\lim _{k \rightarrow \infty} \operatorname{Cov}\left(W_{k}, W_{k+n}\right)=0 \quad \lim _{k \rightarrow \infty} \operatorname{Cov}\left(W_{n}, W_{n+k}\right)=0
$$

for each $n=0,1, \ldots$. Thus, given $\epsilon>0$ there exists $n_{0}=n_{0}(\epsilon)$ such that

$$
\left|\operatorname{Cov}\left(W_{i}, W_{j}\right)\right|<\epsilon \quad\left|\operatorname{Cov}\left(W_{n}, W_{n+k}\right)\right|<\epsilon
$$

if $\min \{i, j\}>n_{0}$ and $k>n_{0}$. Now, take $n>2 n_{0}$, then

$$
\begin{gathered}
\left|\sum_{i=1}^{n-1} \sum_{j=i+1}^{n} \operatorname{Cov}\left(W_{i}, W_{j}\right)\right| \leq\left|\sum_{i=1}^{n_{0}} \sum_{j=i+1}^{2 n_{0}} \operatorname{Cov}\left(W_{i}, W_{j}\right)\right| \\
+\left|\sum_{i=1}^{n_{0}} \sum_{j=2 n_{0}+1}^{n} \operatorname{Cov}\left(W_{i}, W_{j}\right)\right|+\left|\sum_{i=n_{0}+1}^{n-1} \sum_{j=i+1}^{n} \operatorname{Cov}\left(W_{i}, W_{j}\right)\right|
\end{gathered}
$$




$$
\leq\left|\sum_{i=1}^{n_{0}} \sum_{j=i+1}^{2 n_{0}} \operatorname{Cov}\left(W_{i}, W_{j}\right)\right|+\epsilon\left(n-2 n_{0}\right)+\epsilon\left(n-n_{0}-1\right)\left(n-n_{0}\right) / 2 .
$$

Consequently, for any $\epsilon>0$

$$
\lim _{n \rightarrow \infty} \operatorname{Var}\left\{\frac{1}{n} X_{n}\right\}<\epsilon / 2 .
$$

Finally, since $E_{x}\left\{W_{n}\right\} \rightarrow \alpha_{1}$ as $n \rightarrow \infty$ it follows that

$$
\sum_{k=1}^{n} E_{x}\left\{W_{k}\right\} / n \rightarrow \alpha_{1} \text { as } n \rightarrow \infty .
$$

Therefore, for $n$ sufficiently large

$$
P_{x}\left\{\left|\frac{1}{n} X_{n}-\alpha_{1}\right|>\epsilon\right\} \leq P_{x}\left\{\frac{1}{n}\left|\sum_{i=1}^{n}\left[W_{i}-E_{x}\left\{W_{i}\right\}\right]\right|>\epsilon\right\} .
$$

This and (4.1) prove the assertion.

Corollary 4.1: From proposition 4.1 we readily deduce that for each $\epsilon>0$

$$
P_{x}\left\{X_{n} \leq\left(\alpha_{1}+\epsilon\right) n\right\} \rightarrow 1 \text { and } P_{x}\left\{X_{n} \leq\left(\alpha_{1}-\epsilon\right) n\right\} \rightarrow 0
$$

as $n \rightarrow \infty$. Consequently

$$
\lim _{n \rightarrow \infty} P^{n}\left(x, B_{n y}\right)=\left\{\begin{array}{ccc}
1 & \text { if } & y \geq \alpha_{1} \\
0 & \text { if } & y<\alpha_{1} .
\end{array}\right.
$$

Denote by

$$
T(y)=\inf \left\{k ; X_{k}>y\right\}
$$

then for any $x<y$

$$
\begin{aligned}
P_{x}\{T(y) & \leq n\}=P_{x}\left\{X_{n}>y\right\} \\
& =1-P^{n}(x, B) .
\end{aligned}
$$

Proposition 4.2: Under $P_{x}$

$$
\frac{1}{y} T(y) \rightarrow \alpha_{1}^{-1} \text { in probability as } y \rightarrow+\infty \text {. }
$$

Proof: Assume $\alpha_{1}>0$, then we have to show

$$
P_{x}\left\{\left|\frac{1}{y} T(y)-\alpha_{1}^{-1}\right| \leq \epsilon\right\} \rightarrow 1 \text { as } y \rightarrow+\infty
$$

for any $\epsilon>0$. Choose $\epsilon \in\left(0, \alpha_{1}^{-1}\right)$ then from (4.9) we deduce

$$
P_{x}\left\{\left|\frac{1}{y} T(y)-\alpha_{1}^{-1}\right| \leq \epsilon\right\}=P_{x}\left\{X_{\left[\left(\alpha_{1}-1-\epsilon\right) y\right]} \leq y\right\}-P_{x}\left\{X_{\left[\left(\alpha_{1}-1+\epsilon\right) y\right]} \leq y\right\}
$$




$$
=P^{\left[\left(\alpha_{1}^{-1}-\epsilon\right) y\right]}\left(x, B_{y}\right)-P^{\left[\left(\alpha_{1}^{-1}+\epsilon\right) y\right]}\left(x, B_{y}\right)
$$

where, as usual, $[x]$ stands for the integer part of $x$. Since

$$
\frac{y}{\left[\left(\alpha_{1}^{-1}-\epsilon\right)\right]} \geq \frac{\alpha_{1}}{1-\epsilon \alpha_{1}}>\alpha_{1}
$$

it follows from (4.7) that

$$
\lim _{y \rightarrow \infty} P^{\left[\left(\alpha_{1}^{-1}-\epsilon\right) y\right]}\left(x, B_{y}\right)=1
$$

Similarly, when $y \rightarrow \infty$

$$
\frac{y}{\left[\left(\alpha_{1}^{-1}+\epsilon\right) y\right]}<\frac{y}{\left(\alpha_{1}^{-1}+\epsilon\right) y-1} \sim \frac{\alpha_{1}}{1+\epsilon \alpha_{1}}<\alpha_{1}
$$

This and (4.7) imply

$$
\lim _{y \rightarrow \infty} P^{\left[\left(\alpha_{1}^{-1}+\epsilon\right) y\right]}\left(x, B_{y}\right)=0
$$

which proves the proposition.

\section{WEAK CONVERGENCE OF $\left\{T_{n}\right\}$}

In this section we show that a sequence of scale factors $\left\{d_{n}\right\}$ exists such that under $P_{x}$

$$
d_{n} T_{n} \stackrel{d}{\rightarrow} Z
$$

where the r.v. $Z$ has an exponential distribution independent of $x$. But first, we need the following auxiliary result.

Proposition 5.1: Assume that condition (4.1) holds, then

$$
\frac{1-\beta\left(X_{n}\right)}{1-\beta\left(n \alpha_{1}\right)} \stackrel{P}{\rightarrow} \text { as } n \rightarrow \infty
$$

where $\alpha_{1}=E\left\{Y_{1}\right\}$.

\section{Proof: Set}

$$
\beta^{-1}(y)=\inf \{x ; \beta(x)>y\}
$$

then for any $\epsilon \in(0,1)$

$$
P_{x}\left\{\left|\frac{1-\beta\left(X_{n}\right)}{1-\beta\left(n \alpha_{1}\right)}-1\right| \leq \epsilon\right\}=P_{x}\left\{\frac{X_{n}}{n \alpha_{1}} \leq \frac{\beta^{-1}\left(\beta\left(n \alpha_{1}\right)(1-\epsilon)+\epsilon\right)}{n \alpha_{1}}\right\}
$$




$$
-P_{x}\left\{\frac{\beta^{-1}\left(\beta\left(n \alpha_{1}\right)(1+\epsilon)-\epsilon\right)}{n \alpha_{1}}\right\}
$$

Since

$$
\begin{aligned}
& \beta^{-1}\left(\beta\left(n \alpha_{1}\right)(1-\epsilon)+\epsilon\right)>n \alpha_{1} \\
& \beta^{-1}\left(\beta\left(n \alpha_{1}\right)(1+\epsilon)-\epsilon\right)<n \alpha_{1}
\end{aligned}
$$

for all $n=1,2, \ldots$, the assertion now follows from proposition 4.1.

Proposition 5.2: Suppose that (4.1) holds, then

$$
\lim _{n \rightarrow \infty} P_{x}\left\{\left[1-\beta\left(n \alpha_{1}\right)\right] T_{n}>u\right\}=e^{-u}
$$

Proof: Denote by

$$
U_{n}=\left[1-\beta\left(X_{n}\right)\right] T_{n} .
$$

Then taking into account (2.10), we have:

$$
\begin{aligned}
P_{x}\left\{U_{n}>u\right\} & =E_{x}\left(P_{x}\left\{T_{n}>\frac{u}{1-\beta\left(X_{n}\right)} \mid X_{n}\right\}\right) \\
= & E_{x}\left(\left\{\beta\left(X_{n}\right)\right\}^{\left[\frac{u}{1-\beta\left(X_{n}\right)}\right)} .\right.
\end{aligned}
$$

Set

$$
R_{n}(u, w)=\left\{\beta\left(X_{n}(\omega)\right)\right\}^{\left[\frac{u}{1-\beta\left(X_{n}(\omega)\right)}\right]} .
$$

Since the function

$$
h(y)=\exp \left\{\frac{1}{1-\beta(y)} \ln \beta(y)\right\}
$$

is non-decreasing on $R$, it follows that

$$
R_{n}(u, \cdot) \leq R_{n+1}(u, \cdot)
$$

at least (a.s.). From this we readily obtain

$$
\lim _{n \rightarrow \infty} R_{n}(u, \omega)=e^{-u}
$$

at least (a.s.) $P_{x}$. Invoking now the monotone convergence theorem, we deduce from (5.6) that

$$
U_{n} \stackrel{d}{\rightarrow} Z
$$

Finally, write

$$
\left[1-\beta\left(n \alpha_{1}\right)\right] T_{n}=\frac{1-\beta\left(n \alpha_{1}\right)}{1-\beta\left(X_{n}\right)} U_{n}
$$


then the proof of (5.4) follows (5.8), proposition 4.2 and a Slutsky's theorem.

Remark 5.1: One can easily show that the sequence of r.v.'s $\left\{U_{n}\right)_{0}^{\infty}$ has the following properties:

$$
\begin{gathered}
E_{x}\left\{U_{n}\right\}=1 \quad \operatorname{Var}\left\{U_{n}\right\}=E_{x}\left\{\beta\left(X_{n}\right)\right\} \\
E_{x}\left\{U_{n+1} \mid U_{1}, \ldots, U_{n}\right\}=1 .
\end{gathered}
$$

Remark 5.2: The result of the last proposition can be easily extended as follows:

Set

$$
V_{n}=\left[1-\beta\left(n \alpha_{1}\right)\right\} T_{n}
$$

then after some straight forward calculations (see Todorovic and Gani, [7]) one can show that for each $k=1,2, \ldots$

$$
\left(V_{n+1}, \ldots, V_{n+k}\right) \stackrel{d}{\rightarrow}\left(Z_{1}, \ldots, Z_{k}\right)
$$

under $P_{x}$, where $\left\{Z_{k}\right\}_{1}$ is an i.i.d. sequence of r.v.'s with common non-negative exponential distribution of $x$. The sequence also possesses a mixing property.

\section{REFERENCES}

[1] R.E. Barlow and F. Proschan, "Statistical Theory of Reliability and Life Testing", Holt, Rhinehart and Winston, New York (1975).

[2] M. Brown and N.R. Changanty, "On the first passage time distribution for a class of Markov chains”, Ann. Prob. 11, (1983), pp. 1000-1008.

[3] D.J. Daley, "Stochastically monotone Markov chains", Z. Wahrsch. verw. Gebiete 10, (1968), pp. 305-317.

[4] B.V. Gnedenko, "Sur la distribution limite du terme maximum d'une série aléatoire", Ann. Math. 44, (1943), pp. 423-453.

[5] B.V. Gnedenko and A.N. Kolmogorov, "Limit Distributions for Sums of Independent Random Variables", Addison-Wesley, Reading, Massachusetts (1954).

[6] S.I. Resnick, "Stability of maxima of random variables defined on a Markov chain", Adv. Appl. Prob. 4, (1972), pp. 285-295.

[7] P. Todorovic and J. Gani, "A Markov renewal process imbedded in a Markov chain", Stoch. Ann. Appl. 7(3), (1989), pp. 339-353. 


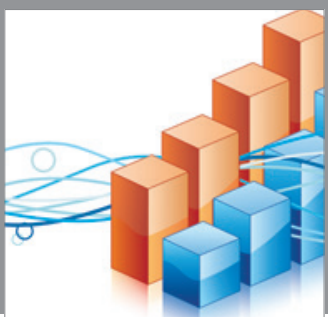

Advances in

Operations Research

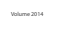

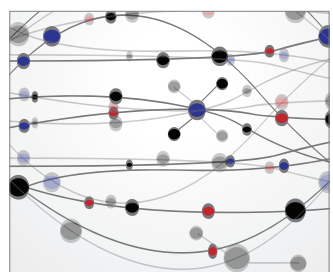

\section{The Scientific} World Journal
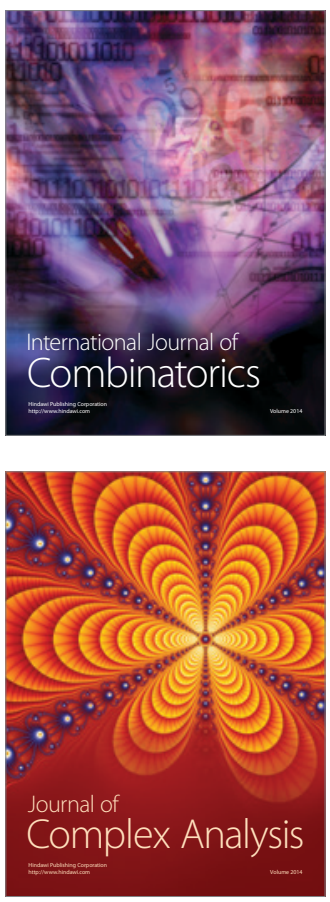

International Journal of

Mathematics and

Mathematical

Sciences
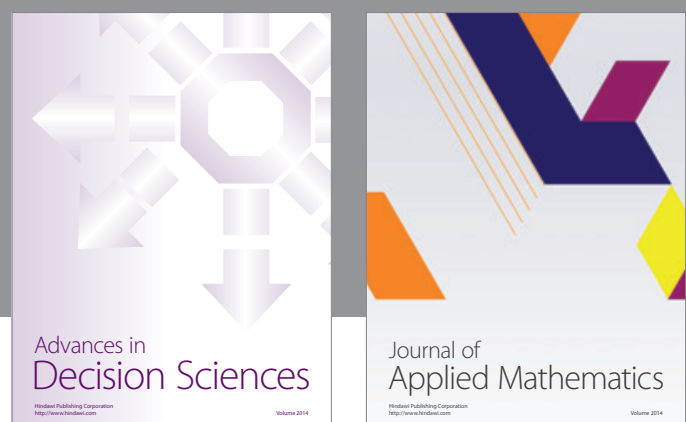

Journal of

Applied Mathematics
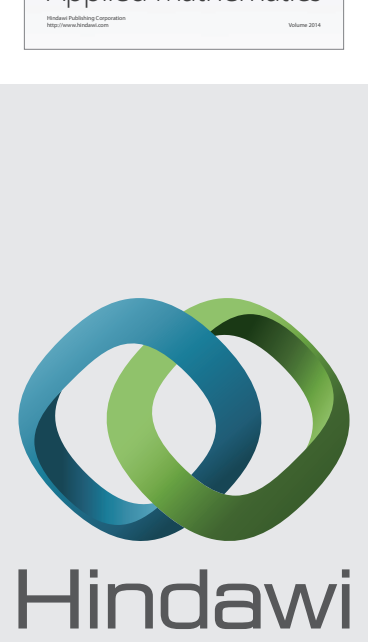

Submit your manuscripts at http://www.hindawi.com
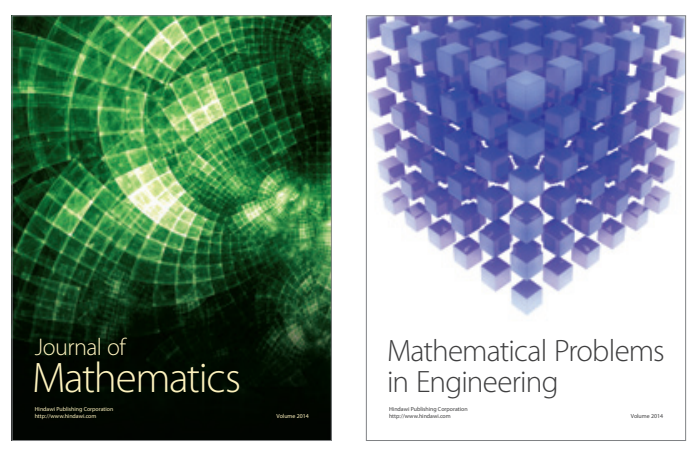

Mathematical Problems in Engineering
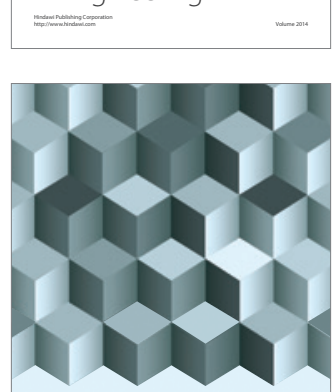

Journal of

Function Spaces
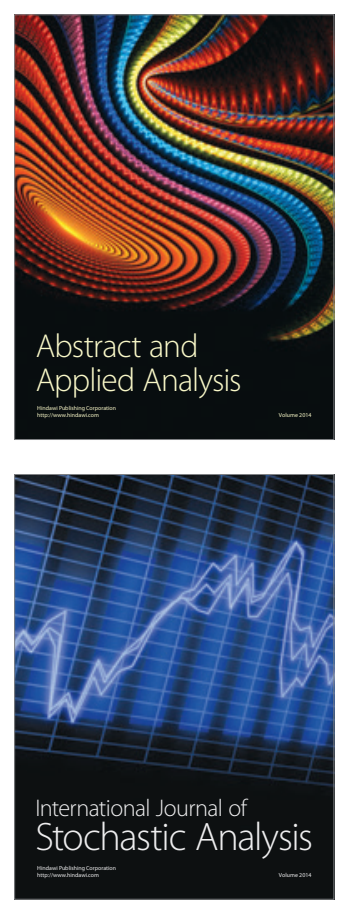

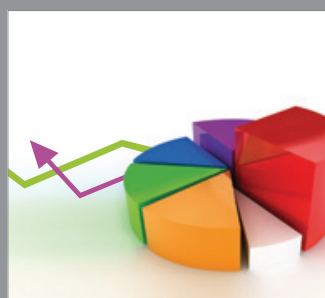

ournal of

Probability and Statistics

Promensencen
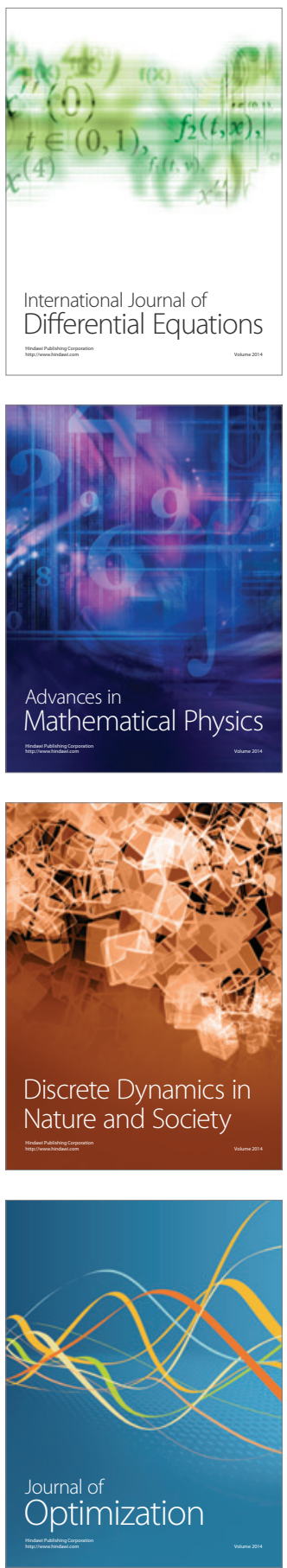